\title{
Favourable outcomes for the first 10 years of kidney and pancreas transplantation at Wits Donald Gordon Medical Centre, Johannesburg, South Africa
}

\author{
J Fabian, ${ }^{1,2}$ MB ChB, FCP (SA), Cert Neph (SA); H Maher, ${ }^{1}$ Registered Nurse; A Bentley, ${ }^{1,3} \mathrm{PhD}$; P Gaylard, ${ }^{4} \mathrm{PhD}$; \\ K Crymble, ${ }^{1}$ Registered Nurse; B Rossi, ${ }^{1}$ MBA, Registered Nurse; L Aucamp, ${ }^{1}$ Registered Nurse; E Gottlich,,${ }^{1,5}$ MB ChB, DCH, FCP Paed \\ (SA); Cert Neph Paed (SA); J Loveland ${ }^{1,6} \mathrm{MB}$ ChB, FCS (SA), Cert Paed Surg (SA); J R Botha, ${ }^{1}$ MB ChB, FCS (SA); \\ J Botha, ${ }^{1} \mathrm{MB}$ ChB, FCS (SA); R Britz, ${ }^{1} \mathrm{MB}$ ChB, FCS (SA) \\ ${ }^{1}$ Wits Donald Gordon Medical Centre, Johannesburg, South Africa \\ ${ }^{2}$ Department of Internal Medicine, Faculty of Health Sciences, University of the Witwatersrand, Johannesburg, South Africa \\ ${ }^{3}$ Department of Family Medicine, Faculty of Health Sciences, University of the Witwatersrand, Johannesburg, South Africa \\ ${ }^{4}$ DMSA (Data Management and Statistical Analysis), Johannesburg, South Africa \\ ${ }^{5}$ Morningside Mediclinic, Johannesburg, South Africa \\ ${ }^{6}$ Department of Paediatric Surgery, Chris Hani Baragwanath Academic Hospital and Faculty of Health Sciences, University of the \\ Witwatersrand, Johannesburg, South Africa
}

Corresponding author: J Fabian (june.fabian@mweb.co.za)

Background. It is important for centres participating in transplantation in South Africa (SA) to audit their outcomes. Wits Donald Gordon Medical Centre (WDGMC), Johannesburg, SA, opened a transplant unit in 2004. The first 10 years of kidney and pancreas transplantation were reviewed to determine outcomes in respect of recipient and graft survival.

Methods. A retrospective review was conducted of all kidney-alone and simultaneous kidney-pancreas (SKP) transplants performed at WDGMC from 1 January 2004 to 31 December 2013, with follow-up to 31 December 2014 to ensure at least 1 year of survival data. Information was accessed using the transplant registers and clinical records in the transplant clinic at WDGMC. The Kaplan-Meier method was used to estimate 1-, 5- and 10-year recipient and graft survival rates for primary (first graft) kidney-alone and SKP transplants.

Results. The overall 10-year recipient and graft survival rates were $80.4 \%$ and $66.8 \%$, respectively, for kidney-alone transplantation. In the kidney-alone group, children tended towards better recipient and graft survival compared with adults, but this was not statistically significant. In adults, recipient survival was significantly better for living than deceased donor type. Recipient and graft survival were significantly lower in black Africans than in the white (largest proportion in the sample) reference group. For SKP transplants, the 10-year recipient survival rate was $84.7 \%$, while kidney and pancreas graft survival rates were $73.1 \%$ and $43.2 \%$, respectively.

Conclusion. Outcomes of the first 10 years of kidney and pancreas transplantation at WDGMC compare favourably with local and international survival data.

S Afr Med J 2016;106(2):172-176. DOI:10.7196/SAMJ.2016.v106i2.10190

Solid-organ transplantation began in South Africa (SA) when the first kidney transplant was performed at the old Johannesburg Hospital on 25 August 1966. ${ }^{[1]}$ In 1973, the only centres offering transplantation were in the public sector, namely Johannesburg General, Baragwanath, Addington, HF Verwoerd and Groote Schuur hospitals. By 1982, Universitas and Tygerberg hospitals had commenced transplantation. ${ }^{[2,3]}$ There are now 18 transplant centres throughout SA, eight in the public sector and ten in the private sector. It is noteworthy that all these centres are clustered in large urban settings and are located in only four of the nine provinces in SA, making access to transplantation relatively inequitable. ${ }^{[4]}$

Wits Donald Gordon Medical Centre (WDGMC), University of the Witwatersrand, Johannesburg, SA, is a private academic teaching hospital. In 2004, a solid-organ transplant unit was established that now offers adult and paediatric kidney, simultaneous kidney-pancreas (SKP), pancreas alone (PA), pancreas after kidney (PAK), liver and combined liver-kidney (CLK) transplantation. Liver, kidney and SKP transplants have been performed since 2004, CLK transplantation began in 2007, a hand-assisted laparoscopic live donor nephrectomy (HALLDN) programme was introduced in 2008 , and pancreas- only transplantation (PA/PAK) has been performed since 2012. A paediatric living-donor liver transplant programme was started in 2013 and is currently the only such programme in southern Africa.

Many international centres that participate in transplantation programmes are obliged to report outcomes annually to a national registry. Unfortunately, the South African Dialysis and Transplant Registry last published national transplant survival data in 1994. The Organ Donor Foundation (ODF) collects annual transplant statistics for SA, but this reporting is voluntary and does not include survival. Outcomes for paediatric liver transplantation in the Johannesburg region have recently been published. ${ }^{[5]}$ An audit of the adult liver transplantation programme in Johannesburg will be published separately. The focus of this article is on reporting recipient and graft survival from kidney and pancreas transplantation at WDGMC during the first 10 years.

\section{Methods}

A retrospective review was conducted of all kidney and pancreas transplants performed at WDGMC from 1 January 2004 to 31 December 2013. Permission to conduct the review was granted by the University of the Witwatersrand Human Research Ethics Committee 
(Medical) (M140647). Data were extracted from regulatory registers and patient records in the transplant clinic, from on-site pathology laboratories for blood results, and from patient records from participating doctors at WDGMC and in clinical practice outside the hospital, all of whom were involved in the long-term care of recipients after transplantation. All the patients who underwent transplants were funded by private medical insurance. Where necessary, clinicians were contacted regarding survival of their patients and the most recent laboratory test results were obtained for the survival analyses.

Data comprised the total number of transplants, repeat transplants, solid organs transplanted, recipient age (adults were defined as $\geq 18$ years), gender, ethnicity, date of transplant, donor type (deceased v. living) and duration of post-transplant follow-up. In the case of deceased donation, all donors were heart-beating and braindead; there was no donation after cardiac death. SKP transplantation was only offered to patients with type 1 diabetes who had been rigorously screened to exclude vascular disease before transplantation.

Kaplan-Meier recipient and graft survival analyses were conducted for transplants carried out during the study period, with at least 1 year of follow-up of all recipients. Those who had died or were lost to follow-up were censored in the graft survival analyses. Data were not censored for death with a functioning graft. Kidney graft survival, for the primary (first) graft only, was determined by the latest available serum creatinine level, and graft loss was defined by the date of commencement of post-transplant chronic dialysis, graft removal, retransplantation or death. Pancreas graft loss was defined by the date of surgical removal of the organ, commencement of insulin therapy if the graft was in situ, or death. Survival analysis for pancreas recipients only included adult SKP transplants, as the numbers of pancreas-only and paediatric pancreas recipients were very small.

Cox proportional hazards regression was used for between-group comparisons for adult v. paediatric recipients, living $\mathrm{v}$. deceased donors, gender and ethnicity. Selfreported ethnicity was categorised as Asian, black African, mixed or white. Because numbers of recipients of mixed ethnicity were small and none of them had reached 10 years of follow-up, these patients were excluded from the between-group ethnicity survival analysis. The $5 \%$ significance level was used throughout and analysis was carried out using SAS version 9.4 (SAS Institute Inc., USA). National statistics were obtained from the ODF annual reports.

Table 1. Demographics and 1-, 5- and 10-year patient and graft survival for primary kidney-alone transplants performed at WDGMC, 2004 - 2013

\begin{tabular}{|c|c|c|c|}
\hline Variable & Overall & 0 - 17 years & $\geq 18$ years \\
\hline Recipients, $N$ & 355 & 50 & 305 \\
\hline Age (years), mean (SD) & & $10.3(4.1)$ & $42.3(12.3)$ \\
\hline \multicolumn{4}{|l|}{ Donor type, $n(\%)$} \\
\hline NRLD & $31(8.7)$ & $2(4.0)$ & $29(9.5)$ \\
\hline RLD & $123(34.6)$ & $30(60.0)$ & $93(30.5)$ \\
\hline Deceased & $201(56.7)$ & $18(36.0)$ & $183(60.0)$ \\
\hline \multicolumn{4}{|l|}{ Gender, $n(\%)$} \\
\hline Female & $130(36.6)$ & $19(38.0)$ & $111(36.4)$ \\
\hline Male & $224(63.1)$ & $30(60.0)$ & $194(63.6)$ \\
\hline Unknown & $1(0.3)$ & $1(2.0)$ & $0(0.0)$ \\
\hline \multicolumn{4}{|l|}{ Ethnicity, $n(\%)$} \\
\hline Asian & $40(11.3)$ & $6(12.0)$ & $34(11.1)$ \\
\hline Black African & $123(34.6)$ & $16(32.0)$ & $107(35.1)$ \\
\hline Mixed & $19(5.4)$ & $3(6.0)$ & $16(5.3)$ \\
\hline White & $156(43.9)$ & $22(44.0)$ & $134(43.9)$ \\
\hline Unknown & $17(4.8)$ & $3(6.0)$ & $14(4.6)$ \\
\hline \multicolumn{4}{|c|}{ 1-year survival, \% (95\% CI) } \\
\hline Patient & $94.2(91.3-96.3)$ & $98.0(86.6-99.7)$ & $93.7(90.3-95.9)$ \\
\hline Kidney & $91.7(88.3-94.1)$ & $93.8(82.0-98.0)$ & $91.3(87.5$ - 94.0) \\
\hline \multicolumn{4}{|c|}{ 5-year survival, \% (95\% CI) } \\
\hline Patient & $87.8(83.4-91.1)$ & $98.0(86.6-99.7)$ & $86.1(81.1-89.9)$ \\
\hline Kidney & $80.3(74.9-84.7)$ & $87.7(72.0-94.9)$ & $79.1(73.1-83.9)$ \\
\hline \multicolumn{4}{|c|}{10 -year survival, \% (95\% CI) } \\
\hline Patient & $80.4(70.5-87.2)$ & $98.0(86.6-99.7)$ & $77.9(67.0-85.5)$ \\
\hline Kidney & $66.8(55.0-76.1)$ & $87.7(72.0-94.9)$ & $64.6(52.1-74.6)$ \\
\hline
\end{tabular}

\section{Results}

During the study period, WDGMC performed $15.2 \%$ (368/2 424) of all kidney transplants and $79.1 \%(72 / 91)$ of all pancreas (PA, PAK, SKP) transplants in SA. Of the kidney-alone recipients, $96.5 \%$ $(355 / 368)$ received a primary graft and $3.0 \%(11 / 368)$ a second graft, two recipients $(0.5 \%)$ receiving a third graft. The total number of paediatric kidney-alone recipients comprised 51/368 (13.9\%), and all except one received primary grafts. After introduction of the HALLDN programme in $2008,73.4 \%(94 / 128)$ of all livingdonor nephrectomies were performed using this technique. Of all the pancreas transplants performed, 93.1\% (67/72) were SKP transplants, 5.5\% (4/72) were PAK transplants and one (1.4\%) was a PA transplant. Only one paediatric SKP transplant occurred during this period.

The demographics and recipient and graft survival rates for all primary kidneyalone transplants are shown in Table 1 .
For primary kidney-alone transplantation, the male/female ratios were 1.6 and 1.8 for children and adults, respectively. In the paediatric group the most common source of the organ was a live donor (64.0\%), while deceased donation predominated in adults (60.0\%).

Overall, the 1-year primary kidneyalone survival rate was $94.2 \%$ for recipients and $91.7 \%$ for grafts. After 10 years, $80.4 \%$ of recipients had survived with a corresponding $66.8 \%$ survival of their grafts. When looking at Kaplan-Meier survival of subgroups within the primary kidney-alone transplants, the paediatric group tended towards better recipient and graft survival compared with adults, but this was not statistically significant. Among the adults, there were no significant gender differences for recipient $(p=0.69)$ or graft $(p=0.40)$ survival. Survival of adult living-donor recipients was significantly better than that of deceased-donor recipients $(p=0.047$; hazard ratio (HR) 0.48 ; $95 \%$ confidence 


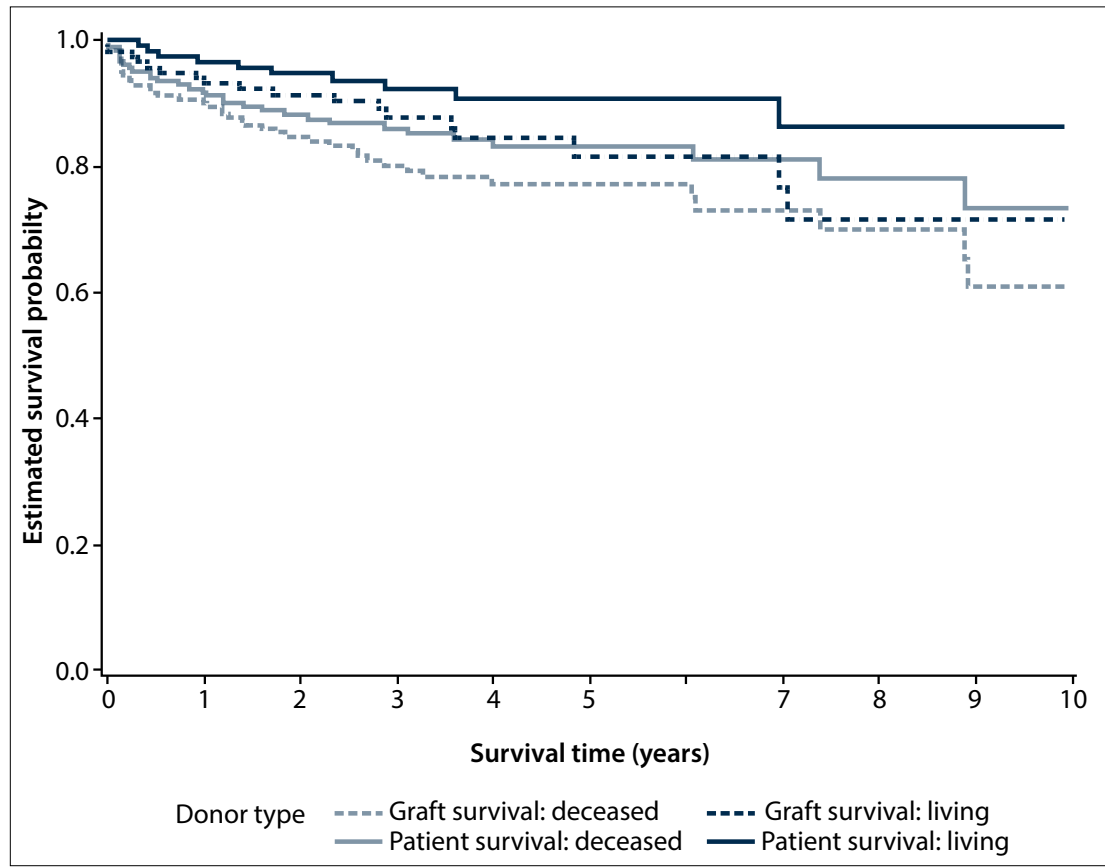

Fig. 1. Kaplan-Meier recipient and graft survival for adult primary kidney-alone transplants at WDGMC, 2004 - 2013 (living v. deceased donor type).

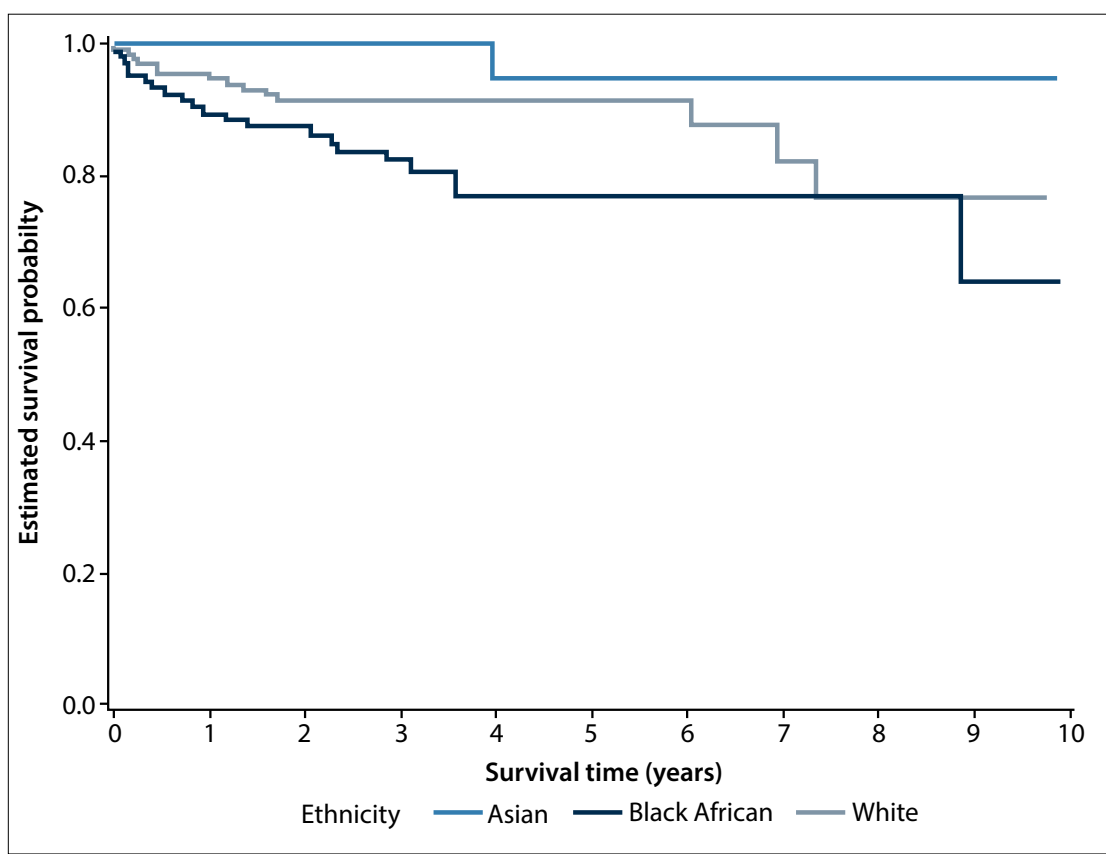

Fig. 2. Kaplan-Meier recipient survival for adult primary kidney-alone transplants at WDGMC, 2004 - 2013 (by ethnicity).

interval (CI) 0.24 - 0.99) (Fig. 1). There was no difference in graft survival when comparing living v. deceased donor type $(p=0.11)$. Survival of adult black recipients was significantly worse than the reference group of white recipients ( $p=0.038$; HR 2.05; 95\% CI 1.04 - 4.03) (Fig. 2). Similarly, kidney graft survival was significantly worse in adult black recipients ( $\mathrm{p}=0.042$; HR 1.78 ; 95\% CI 1.02 - 3.09) and significantly better in Asian recipients $(p=0.035$; HR 0.12; $95 \%$
CI 0.02 - 0.86) compared with the reference group of white recipients (Fig. 3).

The demographics and recipient and graft survival rates for all adult SKP transplants are shown in Table 2. In the adult SKP group $(n=66)$, the male/female ratio was 0.69. Overall, for adult SKP transplants the 10-year recipient, kidney and pancreas survival rates were $84.7 \%, 73.1 \%$ and $43.2 \%$, respectively. The Kaplan-Meier survival curves are depicted in Fig. 4.

\section{Discussion}

Overall, both 1-year recipient and graft survival rates for kidney-alone transplantation at WDGMC were $>90 \%$. The 10-year survival rate for kidney-alone transplantation was $80.4 \%$ for recipients and $66.8 \%$ for grafts. In the adults, recipient survival was better for living-donor recipients, and both recipient and graft survival were worse in the black population. The 10-year survival rates for adult SKP transplantation at WDGMC for recipient, kidney and pancreas were $84.7 \%, 73.1 \%$ and $43.2 \%$, respectively.

Outcomes for adult kidney-alone transplantation at WDGMC compare favourably with international transplant centres. The adult 10 -year graft survival rate $(64.6 \%)$ was similar to that reported for Spain $(71.3 \%)$ in a recent review that compared outcomes in the USA with those in Spain, ${ }^{[6]}$ and better than that reported for the USA (53.4\%). Similarly, 10-year recipient survival (77.9\%) was comparable to that in Spain (86.2\%) and superior to that in the USA $(67.4 \%){ }^{[6]}$

In the adult primary kidney transplant group, the significantly worse outcomes for black recipients and their grafts paralleled outcomes seen in African Americans. Deceased donor graft survival in African Americans has been shown to be significantly worse than that observed for other ethnic groups (Europeans, Hispanics and white Americans), and this difference was accentuated 5 and 10 years after transplantation. ${ }^{[7]}$ Our results, although similar to those for African Americans at 5 and 10 years after transplantation, contradict findings from a study at Tygerberg Hospital in Cape Town, where no significant difference in graft survival based upon ethnicity was found. However, the period under review at Tygerberg was earlier than in this study and there were far fewer black recipients $\left(n=56 / 542(10.3 \%) .{ }^{[8]}\right.$

A host of factors have been proven to predispose African Americans to poorer graft outcomes. For example, Medicare health funder policy only finances immunosuppressant medication for the first 3 years after transplantation, which forces patients to buy their own expensive medication thereafter. Consequently compliance falls, contributing to graft loss. African Americans have lower rates of living-related donation, and higher rates of immunological rejection and HLA mismatching, and persistent hypertension after the transplant. ${ }^{[9,10]}$ Recently identified genetic mutations in the APOL1 gene in African American living donors may also impact adversely on graft function after donation. ${ }^{[11,12]}$ Our preliminary findings 


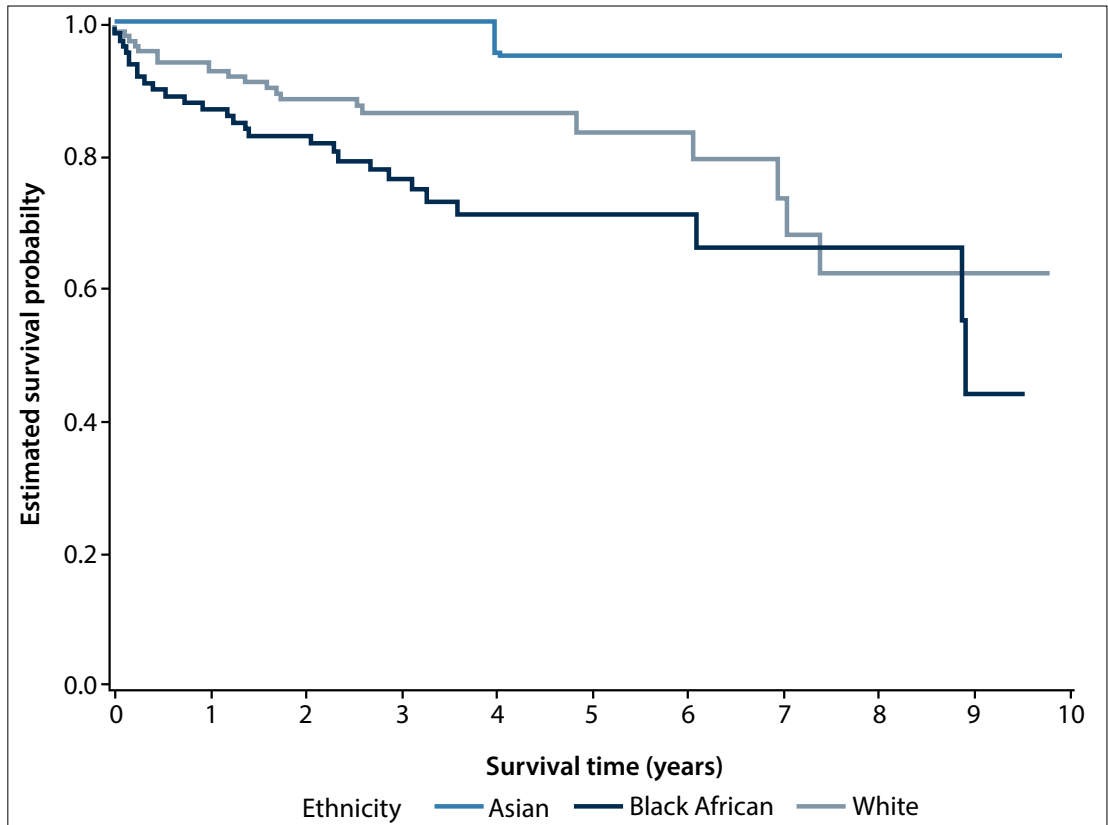

Fig. 3. Kaplan-Meier graft survival for adult primary kidney-alone transplants at WDGMC, 2004 2013 (by ethnicity).

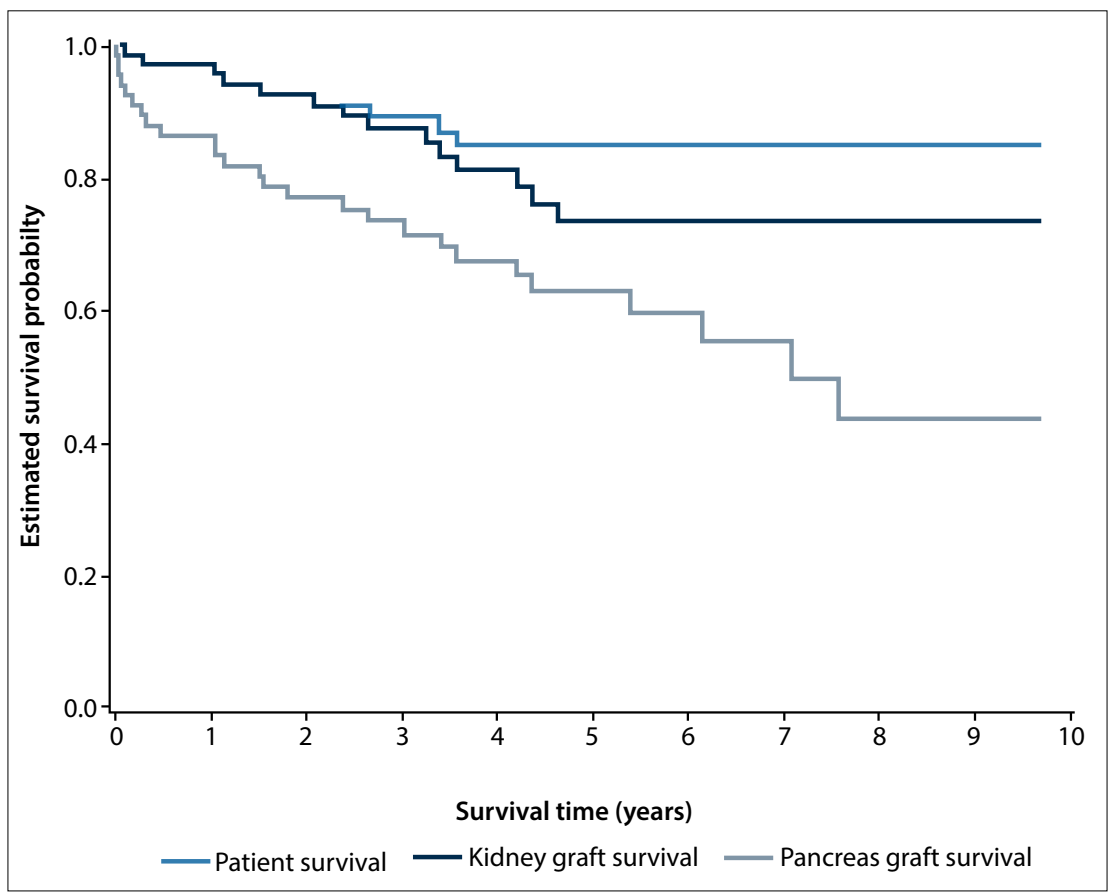

Fig. 4. Kaplan-Meier recipient and graft survival for SKP transplants at WDGMC, 2004 - 2013.

highlight the need for rigorous research into the SA kidney transplant donor and recipient community in order to determine population-specific factors that influence outcomes.

The paediatric primary kidney recipient and graft survival in this cohort was excellent, with $>90 \%$ recipient and $>85 \%$ graft survival at 10 years. However, the estimates are not very precise because of the small number of children who have had transplants so far. Charlotte Maxeke
Johannesburg Academic Hospital (CMJAH) reviewed 282 paediatric transplants from 1984 to $2003 .^{[13]}$ Ten-year recipient and graft survival rates were $68 \%$ and $23 \%$, respectively. Red Cross War Memorial Children's Hospital (RCWMCH) in Cape Town reviewed 89 transplants from 1995 to 2005 and found the 7-year graft survival rate to be $72 \% .{ }^{[14]}$ In a recent publication from the USA that reviewed 17000 paediatric kidney transplants, outcomes were significantly better after 2001, which may be related
Table 2. Demographics and 1-, 5- and 10 -year patient and graft survival for SKP transplants performed at WDGMC in adults, 2004 - 2013

Variable

Gender, $n$ (\%)

Female

39 (59.1)

Male

27 (40.9)

Ethnicity, $n$ (\%)

Asian

Black African

Mixed

White

Unknown $6(9.1)$

Age (years) mean (SD) $34.6(6.1)$

1-year survival, \% (95\% CI)

Patient

$97.0(88.4-99.2)$

Kidney $97.0(88.4-99.2)$

Pancreas $86.1(75.0$ - 92.5)

5-year survival, \% (95\% CI)

Patient

$84.7(72.5-91.8)$

Kidney $73.1(58.2-83.5)$

Pancreas $62.6(48.5-73.8)$

10-year survival, \% (95\% CI)

Patient

84.7 (72.5 - 91.8)

Kidney

Pancreas $43.2(24.5-60.6)$

to better immunosuppression, with 10-year recipient and graft survival rates of $90.5 \%$ and $60.2 \%$, respectively. ${ }^{[15]}$ Explanations for the relatively good survival in our cohort, given that it is still a small sample, may reflect the more 'recent' time frame of analysis; higher living donor rates (64\%) compared with $24 \%$ at RCWMCH and $21 \%$ at CMJAH; fewer black children, who have been shown to have poorer graft survival; fewer repeat transplants; and the assumption that socioeconomic circumstances are better in patients accessing a funded healthcare environment. ${ }^{[16]}$

SKP transplantation is considered the treatment of choice for type 1 diabetics with end-stage kidney disease who are $<50$ years of age. ${ }^{[17,18]}$ There is, however, a debate around this in the literature, because some centres have shown inferior survival for SKP transplantation when compared with living donor kidney-alone transplantation in type 1 diabetics with end-stage kidney disease. ${ }^{[19]}$ Unfortunately 
we do not have sufficient numbers of type 1 diabetics who received a kidney-alone transplant to compare their outcomes with the SKP group, but adult recipient and kidney graft survival rates in the SKP group were comparable to those in the kidney-alone group (Table 2). Pancreas survival at 5 years is commonly $>60 \%$, and this was also observed in this series. ${ }^{[20]}$ Ten-year survival data, although more scarce, are generally $>50 \%$, which is in line with this cohort at $43.2 \%$ (95\% CI $24.5-60.6)$.

\section{Study limitations}

There are limitations to this study. Firstly, the annual national statistics from the ODF rely on voluntary reporting of transplant activity from individual transplant units. Although the ODF is the only available source of national transplant statistics, these may not accurately reflect transplant activity if units do not submit data, or submit incomplete or inaccurate data. Annual reports are compiled and returned to participating units after data submission. There were some minor discrepancies when comparing data from the WDGMC registers with ODF national statistics. The transplant statistics in this article were from the WDGMC registers/recipient records in preference to ODF statistics, as the former have been accurately verified.

Secondly, the numbers of patients who underwent transplants in this series were relatively small and this prevented us from doing further analyses to understand some of the findings, for example exploring the observed differences in outcomes for black v. white recipients and their grafts. Unfortunately no further analysis of deceased v. living donor graft survival in black recipients was possible owing to the small numbers of patients at 5 and 10 years after the transplant. This also limited comparisons with larger cohorts internationally.

\section{Conclusion}

Outcomes of kidney and pancreas transplantation for the first 10 years at WDGMC compare favourably with local and international data. Further research is required into the SA donor and recipient pool to identify specific risk factors that could be addressed to improve recipient and graft survival.
Acknowledgements. We thank the dedicated team in the transplant clinic at WDGMC, the doctors and their staff who willingly volunteered clinical information regarding the long-term survival of their patients after transplantation, the patients who have undergone transplantation at WDGMC, the donors and families who generously donated organs for transplantation, and Mr Masupha Adoro for data capture.

\section{References}

1. Schreiber L, Gillwald J. Johannesburg Hospital/Hospitaal 1890-1990: History of the Hospital. Johannesburg: Johannesburg Hospital Board, 1990.

2. Furman K. Maintenance haemodialysis and renal transplantation facilities in South Africa July 1974. S Afr Med J 1975;49(11):394-396.

3. Furman K, du Toit E. Facilities for treatment and patients on renal replacement therapy in South Africa: A report on the 1982 registration. S Afr Med J 1983;63(11):407-408.

4. Organ Donor Foundation of South Africa. 2014. https://www.odf.org.za/ (accessed 6 November 2014).

5. Loveland J, Britz R, Joseph C, et al. Paediatric liver transplantation in Johannesburg revisited: 59 transplants and challenges met. S Afr Med J 2014;104(11):799-802. [http://dx.doi.org/10.7196/SAMJ.8627]

6. Ojo AO, Morales JM, Gonzalez-Molina M, et al. Comparison of the long-term outcomes of kidney transplantation: USA versus Spain. Nephrol Dial Transplant 2013;28(1):213-220. [http://dx.doi.org/10.1093/ndt/gfs287]

7. Gondos A, Döhler B, Brenner H, Opelz G. Kidney graft survival in Europe and the United States: Strikingly different long-term outcomes. Transplantation 2013;95(2):267-274. [http://dx.doi.
[ Strikingly different long-term o
org/10.1097/TP.0b013e3182708ea8]

8. Moosa MR. Impact of age, gender and race on patient and graft survival following renal transplantMoosa MR. Impact of age, gender and race on patient and graft survivat
ation - developing country experience. S Afr Med J 2003;93(9):689-695.

9. Katznelson S, Gjertson D, Cecka J. The effect of race and ethnicity on kidney allograft outcome. Clin Transpl 1995:379-394

10. Cosio FG, Dillon JJ, Falkenhain ME, et al. Racial differences in renal allograft survival: The role of systemic hypertension. Kidney Int 1995;47(4):1136-1141. [http://dx.doi.org/10.1038/ki.1995.162]

11. Cohen DM, Mittalhenkle A, Scott DL, Young CJ, Norman DJ. African American living-kidney donors should be screened for APOL1 risk alleles. Transplantation 2011;92(7):722-725. [http://dx.doi. org/10.1097/TP.0b013e31822

12. Reeves-Daniel AM, DePalma JA, Bleyer AJ, et al. The APOL1 gene and allograft survival after kidney transplantation. Am J Transplant 2011;11(5):1025-1030. [http://dx.doi.org/10.1111/j.1600-6143.2011.03513.x]

13. Pitcher GJ, Beale PG, Bowley DM, Hahn D, Thomson PD. Pediatric renal transplantation in a South African teaching hospital: A 20-year perspective. Pediatr Transplant 2006;10(4):441-418. [http:// dx.doi.org/10.1111/j.1399-3046.2006.00489.x].

14. McCulloch M, Gajjar P, Spearman C, et al. Overview of a paediatric renal transplant programme. S Afr Med J 2008;96(9):955-959.

15. Van Arendonk KJ, Boyarsky BJ, Orandi BJ, et al. National trends over 25 years in pediatric kidney transplant outcomes. Pediatrics 2014;133(4):594-601. [http://dx.doi.org/10.1542/peds.2013-2775]

16. Patzer RE, Mohan S, Kutner N, McClellan WM, Amaral S. Racial and ethnic disparities in pediatric renal allograft survival in the United States. Kidney Int 2015;87(3):584-592. [http://dx.doi.org/10.1038/ki.2014.345] 17. Waki K, Terasaki PI, Kadowaki T. Long-term pancreas allograft survival in simultaneous pancreaskidney transplantation by era: UNOS registry analysis. Diabetes Care 2010;33(8):1789-1791. [http:// dx.doi.org/10.2337/dc09-2276]

18. Ojo AO, Meier-Kriesche H-U, Hanson JA, et al. The impact of simultaneous pancreas-kidney transplantation on long-term patient survival. Transplantation 2001;71(1):82-89.

19. Morath C, Zeier M, Döhler B, et al. Transplantation of the type 1 diabetic patient: The long-term benefit of a functioning pancreas allograft. Clin J Am Soc Nephrol 2010;5(3):549-552. [http://dx.doi. org/10.2215/CJN.03720609]

20. Gruessner RW, Gruessner AC. The current state of pancreas transplantation. Nature Reviews Endocrinology 2013;9(9):555-562. [http://dx.doi.org/10.1038/nrendo.2013.138]

Accepted 19 October 2015. 